\title{
Synchronization and a secure communication scheme using optical star network
}

\begin{abstract}
This work aims to show the effect of synchronization phenomena in multi-nodal star optical network topology as well as to develop an efficient symmetric cryptosystem utilizing available parameters. The optical network is based on chaotic semiconductor laser (SL) systems described by dimensionless modified Lang-Kobayashi's (L-K) delay differential equations. The network nodes are mutually connected with a central semiconductor laser hub with bidirectional linear optical feedback. It has been observed that the laser output can be modulated using a star network setup. The laser intensity increases with the number of nodes and its much more higher than the same for solitary laser, keeping all other inputs as constant. So the network topology is an effective way to optimize the output power. The process by each nodes into the network is illustrated graphically for three, five and seven SLs, respectively. Also the whole network can be implemented as an optical communication system for transmission of signals. Each SL can act as a transceiver during communication. The communication process is examined using a chaotic signal as a plaintext connected with the SL hub and successfully provided a symmetrically secure mechanism upon the communication protocol. The result shows the optimization of output power with the increment of the number of nodes. Also the communication scheme can successfully decode the encrypted signal from SL Hub, at each other nodes.
\end{abstract}

Keyword: Semiconductor laser; Symmetrically secured optical communication; Time delayed system. 